\title{
RESEARCH HIGHLIGHT COVID-19: treating and managing severe cases
}

\author{
Jingwen $\mathrm{Ai}^{1}$, Yang $\mathrm{Li}^{1}$, Xian Zhou ${ }^{1}$ and Wenhong Zhang ${ }^{1}$ \\ Cell Research (2020) 30:370-371; https://doi.org/10.1038/s41422-020-0329-2
}

\begin{abstract}
The recent outbreak of COVID-19 caused by SARS-CoV-2 has led to a global pandemic, but an international consensus on its treatment is still unavailable. In this highlight, we illustrate current strategies for the management of severe COVID-19 patients, and discuss the potential utility of antiviral drugs and glucocorticoids.
\end{abstract}

The treatment of severe and critical COVID-19 patients is of grave concern due to the high mortality of the disease. Therefore, the first step is to closely monitor the mild patients to prevent disease exacerbation. The clinical manifestations, vital signs, blood oxygen saturation, fluid volume, gastrointestinal tract function and mental state of all patients should be dynamically monitored every day. In addition to routine testing of laboratory parameters, some potential indicators for severe cases should also be monitored and regularly followed up for testing, including lactate dehydrogenase, D-dimer, IL-6, peripheral blood lymphocyte count and $\mathrm{CD}^{+}{ }^{+}$T lymphocytes, etc. ${ }^{1,2}$ For patients with high risk of severe illness, anticoagulation treatment with heparin is recommended. Low molecular weight heparin (LMWH) should be maintained at 1 to 2 doses per day until the level of $D$-dimer returns to normal. For conditions of $\mathrm{FDP} \geq 10 \mu \mathrm{g} / \mathrm{mL}$ and/or D-dimer $\geq 5 \mu \mathrm{g} / \mathrm{mL}$, unfractionated heparin is recommended.

For severe and critical cases, comprehensive and refined management should be adopted. Severe patients are currently found to have systemic multiple-organ disfunction. ${ }^{3-5}$ Therefore, on the basis of sufficient respiratory support in strict accordance with national diagnosis and treatment guidelines, attention should also be paid to the support and protection of circulation, kidney and intestinal function. For patients who may have a "cytokine storm", intermittent short-term hemofiltration and immunomodulatory therapy may be an option. Clinical microbiological testing is required for critical patients, as well as the follow-up of procalcitonin and C-reactive protein; patients suspected for bacterial or fungal infections should have pathogen microbiological culture in time, and molecular diagnostic tests of pathogens, including PCR-based molecular biology testing and next-generation sequencing.

In terms of prevention and control of hospital-acquired infection, it is necessary to strictly abide by the relevant technical guidelines, ${ }^{6,7}$ strengthen process management, and correctly select and use personal protective equipment such as masks, isolation suits, protective clothing, eye masks, protective masks, gloves, etc. The implementation of various disinfection and isolation measures minimizes the risk of hospital infections and eliminates hospitalacquired infection by medical staff.

Antiviral treatment: At present, the efficacy of antiviral drugs still needs to be evaluated through further clinical studies. Most of the existing studies are single-arm studies or studies with a small sample size. Existing data suggest that lopinavir/ritonavir treatment shows no statistically significant difference in 28-day mortality and viral clearance in severe cases of COVID-19, but in the modified intention-to-treat analysis, the lopinavir/ritonavir group had a significantly shorter length of ICU stay and higher 14day clinical improvement rate than the control group. ${ }^{8}$ Therefore, the current study suggested that the antiviral efficacy of lopinavir/ ritonavir might not be significant.

Multiple studies on hydroxychloroquine sulfate or chloroquine phosphate are currently being conducted in several countries. An early study in France suggested that in 6 asymptomatic nucleic acidpositive patients, the treatment of hydroxychloroquine and azithromycin resulted in a $100 \%$ negative conversion rate of SARSCoV-2 nucleic acid from the nasopharyngeal swabs. But the sample size of this study was too small to obtain statistical results between the groups and this has caused controversial opinions. ${ }^{9}$ A recently released study involving more than a thousand patients in IHU Méditerranée Infection, Marseille, France suggested that the disease progression rate of patients using hydroxychloroquine + azithromycin was $4.3 \%$, and the mortality rate among these patients was $0.47 \%$ (http://www.mediterranee-infection.com/wp-content/ uploads/2020/04/Abstract_Raoult_EarlyTrtCovid19_09042020_vD1v. pdf). The mortality rate seemed relatively lower compared to that previously reported for COVID-19 cases, but the study also lacked a control group and hasn't yet gone through the peer review process. More studies on the efficacy of hydroxychloroquine + azithromycin will still be needed.

Remdesivir is an RNA-dependent RNA polymerase inhibitor with broad-spectrum activity against various virus families. The latest compassionate use of Remdesivir for patients with severe COVID-19 found that after Remdesivir treatment, $68 \%$ of 53 patients had remission of symptoms and the mortality rate was about $18 \% .{ }^{10}$ The mortality rate of severe cases reported in this study was lower than that in previous reports, but the study has defects such as single-arm design, short follow-up time, and failure to collect quantitative viral load data. Therefore, it is still necessary to accumulate more data to allow for a more comprehensive assessment of the potential utility of the drug.

In short, there is currently no international consensus on antiviral treatment options for COVID-19, and more prospective clinical studies are still needed in the future for further evaluation. Meanwhile, we would need to pay close attention to the possible adverse reactions of various antiviral drugs.

Glucocorticoids: The use of glucocorticoids is an issue of great debate. Based on retrospective analyses of the previous use of glucocorticoids in SARS, H1N1 and other viral pneumonia, the routine use of glucocorticoids is not supported by the World Health Organization (WHO). ${ }^{11}$ However, in clinical practice, glucocorticoids tend to be more frequently used in severe or

${ }^{1}$ Department of Infectious Diseases, Huashan Hospital, Fudan University, Shanghai 200040, China

Correspondence: Wenhong Zhang (zhangwenhong@fudan.edu.cn)

Published online: 29 April 2020 
critical illness. Therefore, if the confounding factors are not adjusted, it is difficult to scientifically evaluate the role of glucocorticoids.

Based on our treatment experience, glucocorticoids can be used in short-term, low-dose applications in patients who are at a high risk of disease progression. ${ }^{12}$ There are two lines of important information here. The first point is "high risk of disease progression". The mechanism of glucocorticoids is to suppress excessive immune responses. In most mild patients, the immune response against the virus is beneficial. The use of glucocorticoids may reduce the clearance of the virus; while for severe or critically ill patients who have already produced a "cytokine storm", the use of glucocorticoids is too late to block this excessive inflammatory response. Therefore, glucocorticoids should be used in the early stage of excessive inflammatory reactions to achieve the best results. According to our experience, clinical manifestations of this period are characterized by rapid radiography progression with or without deterioration of oxygenation levels. That is to say, for patients with mild disease, if there is obvious progression of lung lesions within $48 \mathrm{~h}$, the application of glucocorticoids can be considered to prevent the serious development of these patients. Another key point is "low-to-moderate-dose, short-course". In the case of early application for treating excessive inflammation, the use of low-to-moderate-dose, short-course glucocorticoids might be effective. According to our experience of treating COVID-19 patients at Shanghai, the dose of methylprednisolone was 40-80 $\mathrm{mg} /$ day for 3 days, which was then tapered to $20 \mathrm{mg} /$ day, with a total treatment period of less than 7 days. With this dose of treatment, the side effects and adverse reactions of glucocorticoids are very limited, with satisfactory safety profiles.

\section{ADDITIONAL INFORMATION}

Competing interests: The authors declare no competing interests.

\section{REFERENCES}

1. Zhou, F. et al. Lancet 395, 1054-1062 (2020).

2. Wu, C. et al. JAMA Intern. Med. https://doi.org/10.1001/jamainternmed.2020.0994 (2020).

3. Wang, D. et al. JAMA 323, 1061 (2020).

4. Shi, S. et al. JAMA Cardiol. https://doi.org/10.1001/jamacardio.2020.0950 (2020).

5. Yang, X. et al. Lancet Respir. Med. https://doi.org/10.1016/S2213-2600(20)30079-5 (2020).

6. The National Health Commission of the People's Republic of China. Guidelines for the use of common medical protective equipment in the prevention and control of pneumonia caused by new coronavirus. http://www.nhc.gov.cn/yzygj/s7659/ 202001/e71c5de925a64eafbe1ce790debab5c6.shtml.

7. The National Health Commission of the People's Republic of China. Technical guidelines for the prevention and control of new coronavirus infections in medical institutions (first edition). http://www.nhc.gov.cn/yzygj/s7659/202001/ b91fdab7c304431eb082d67847d27e14.shtml.

8. Cao, B. et al. N. Engl. J. Med. https://doi.org/10.1056/NEJMoa2001282 (2020).

9. Gautret, P. et al. Int. J. Antimicrob. Agents https://doi.org/10.1016/j. ijantimicag.2020.105949 (2020).

10. Grein, J. et al. N. Engl. J. Med. https://doi.org/10.1056/NEJMoa2007016 (2020).

11. Russell, C. D. et al. Lancet 395, 473-475 (2020).

12. Shang, L. et al. Lancet 395, 683-684 (2020). 